\title{
Replica symmetry breaking related to a general ultrametric space I: replica matrices and functionals
}

\author{
A.Yu.Khrennikov, S.V.Kozyrev ${ }^{\dagger}$
}

April 18, 2018

\begin{abstract}
Family of replica matrices, related to general ultrametric spaces, is introduced. These matrices generalize the known Parisi matrices. Some functionals of replica approach are computed.
\end{abstract}

\section{Introduction}

One of the most interesting phenomenons of replica theory of spin glasses and other disordered systems is the property of ultrametricity of the replica space [1], 22. In papers [3] and [4 it was shown that, in important particular case, this ultrametric structure of replica space can be described with the help of $p$-adic analysis, and the Parisi replica matrix, which is the crucial ingredient of the replica approach, can be considered as a $p$-adic pseudodifferential operator. For other discussions on the relation of replicas with analysis on ultrametric groups see also [5]. For general introduction to the replica method see [2].

In papers [6] - 8] a very general family of ultrametric spaces was constructed, and a theory of ultrametric pseudodifferential operators (or PDO) was developed. The bases of ultrametric wavelets in spaces of functions on the constructed ultrametric spaces were introduced, and it was shown that the ultrametric PDO are diagonal in the bases of ultrametric wavelets. This theory is a very far generalization of the results of the papers [9, [10], where the corresponding constructions for the $p$-adic case were introduced.

The mentioned above results are related to the field of $p$-adic and ultrametric mathematical physics. For the other developments in this field see [11]-18]. Among applications of $p$-adic mathematical physics we would like to mention the application to dynamics of macromolecules [19].

\footnotetext{
*International Center for Mathematical Modelling in Physics and Cognitive Sciences, University of Växjö, S-35195, Sweden, e-mail: Andrei.Khrennikov@msi.vxu.se

†Steklov Mathematical Institute, Moscow, Russia, e-mail: kozyrev@mi.ras.ru
} 
In the present paper we, developing the approach of [3] and [4], and propose a new replica symmetry breaking scheme, based on a new family of replica block matrices, related to the general family of ultrametric spaces and ultrametric PDO, considered in [6]- 7].

We introduce a family of replica matrices, related to ultrametric PDO of [6]-[7], and compute for these matrices some functionals of the replica approach.

The organization of the present paper is as follows.

In Section 2 we describe parameterization of the Parisi matrices by abelian groups with ultrametric.

In Section 3 we introduce the new family of replica block matrices.

In Section 4 we show the relation between the introduced replica matrices and restrictions of the ultrametric pseudodifferential operators of [ 6] - 7] on finite dimensional spaces of test functions.

In Section 5 we compute some functionals of the replica approach for introduced replica matrices.

In Section 6 we compare the results of computations of the previous Section with the Parisi replica symmetry breaking scheme.

In Section 7 we put necessary material from [6]-7] on ultrametric spaces and pseudodifferential operators on these spaces.

\section{The Parisi matrices and their parameterization}

The Parisi replica matrix $\left(Q_{a b}\right)$ is the $n \times n$ matrix, defined as follows

$$
\begin{gathered}
Q_{a a}=0 \\
Q_{a b}=q_{i} \quad \text { if } \quad\left[\frac{a}{m_{i}}\right] \neq\left[\frac{b}{m_{i}}\right], \quad\left[\frac{a}{m_{i+1}}\right]=\left[\frac{b}{m_{i+1}}\right]
\end{gathered}
$$

Here $m_{i}$ are integers such that $p_{i}=m_{i+1} / m_{i}$ are integers,

$$
1=m_{0}<m_{1}<\ldots<m_{k}<m_{k}=n
$$

and $q_{i}$ are $k$ real parameters, $[x]$ is the function of $x$, which is equal to the smallest integer greater of equal to $x$.

In [3], 4], it was shown that, for the particular case when $m_{i}=p^{i}$ (i.e. all $p_{i}=p$ ), formula (11) is equivalent to the following formula of the $p$-adic parameterization: after the corresponding renumbering of the indices of the Parisi replica matrix, the matrix elements of this matrix can be put into the form

$$
Q_{a b}=q\left(|a-b|_{p}\right)
$$

where $|\cdot|_{p}$ is the $p$-adic distance and where the function $q(x)$ encodes the information about the matrix elements in the following way: $q\left(p^{i}\right)=q_{i}, q(0)=0$.

Therefore the definition of this particular family of the Parisi matrices is related to the simplest ultrametric spaces - the fields of $p$-adic numbers. 
Let us generalize the construction of papers [3], 4] (of the $p$-adic parameterization of the Parisi matrix) onto the case of general Parisi matrices (see also [5] for the discussion of diagonalization of the Parisi matrices by related construction). Consider the abelian group (and ultrametric space), which is the direct product of finite abelian cyclic groups:

$$
G=\prod_{i=1}^{k} \mathbf{Z} / p_{i} \mathbf{Z}
$$

where $\mathbf{Z}$ is the group of integers and $p_{i}$ are natural numbers. Elements of $G$ has the form $x=\left\{x_{i}\right\}=\left(x_{1}, \ldots, x_{k}\right), 0 \leq x_{i} \leq p_{i}-1$, and the addition is elementwise modulo $p_{i}$ :

$$
x+y=\left\{\left(x_{i}+y_{i}\right) \bmod p_{i}\right\}
$$

Lemma 1 The elements of $G$ are in one to one correspondence with natural numbers in $\left\{1,2, \ldots, \prod_{j=1}^{k} p_{j}\right\}$. This correspondence is given by the map

$$
\begin{gathered}
l: 1,2, \ldots, \prod_{j=1}^{k} p_{j} \rightarrow G \\
l^{-1}: \quad x=\left(x_{1}, \ldots, x_{k}\right) \mapsto 1+\sum_{i=1}^{k} x_{i} \prod_{j=1}^{i-1} p_{j}, \quad 0 \leq x_{i} \leq p_{i}-1
\end{gathered}
$$

Here we define $\prod_{j=1}^{0} p_{j}=1$.

Proof It is easy to see that the map $l^{-1}$ maps 0 in the group $G$ into 1 . Also the image of

$$
\left(p_{1}-1, \ldots, p_{k}-1\right)
$$

is

$$
1+\sum_{i=1}^{k}\left(p_{i}-1\right) \prod_{j=1}^{i-1} p_{j}=1+\sum_{i=1}^{k}\left(\prod_{j=1}^{i} p_{j}-\prod_{j=1}^{i-1} p_{j}\right)=\prod_{j=1}^{k} p_{j}
$$

The images of elements of the group $G$ will lie between 1 and $\prod_{j=1}^{k} p_{j}$.

Check that the map $l^{-1}$ (which we call the enumeration map) maps the different elements of the group $G$ into the different numbers in $\left\{1,2, \ldots, \prod_{j=1}^{k} p_{j}\right\}$.

Let $x \neq y$ be elements of the group $G$ such that $x_{i} \neq y_{i}, x_{j}=y_{j}$ for $j>i$. Then

$$
l^{-1}(x)-l^{-1}(y)=\sum_{j=1}^{i}\left(x_{j}-y_{j}\right) \prod_{l=1}^{j-1} p_{l}=\sum_{j=1}^{i-1}\left(x_{j}-y_{j}\right) \prod_{l=1}^{j-1} p_{l}+\left(x_{i}-y_{i}\right) \prod_{l=1}^{i-1} p_{l}
$$

The module of the sum $\sum_{j=1}^{i-1}\left(x_{j}-y_{j}\right) \prod_{l=1}^{j-1} p_{l}$ is not larger than $\prod_{l=1}^{i-1} p_{l}-1$. The module of the contribution $\left(x_{i}-y_{i}\right) \prod_{l=1}^{i-1} p_{l}$ is not less that $\prod_{l=1}^{i-1} p_{l}$, thus the expression above is not equal to zero. This implies that $l^{-1}$ maps the different elements of $G$ into the different numbers. This finishes the proof of the lemma.

The group $G$ is ultrametric space with the ultrametric $|x|$ defined as

$$
|x|=m_{i}=\prod_{j=1}^{i} p_{j}
$$


where $i$ is the number of the last nonzero $x_{i}$.

We consider the Parisi matrix $Q=\left(Q_{a b}\right)$ as the operator in the space of functions on the group $G$ with the elements (4). The following theorem takes place.

Theorem 2 The matrix element $Q_{a b}$, defined by (1), depends only on the ultrametric of the difference of $l(a)$ and $l(b)$ :

$$
Q_{a b}=q(|l(a)-l(b)|),
$$

where $q\left(m_{i}\right)=q_{i}, q(0)=0, i=1, \ldots, k$.

Proof The condition $\left[\frac{a}{m_{i}}\right]=\left[\frac{b}{m_{i}}\right]$ takes the form

$$
\left[\frac{1+\sum_{i=1}^{k} a_{i} \prod_{j=1}^{i-1} p_{j}}{m_{i}}\right]=\left[\frac{1+\sum_{i=1}^{k} b_{i} \prod_{j=1}^{i-1} p_{j}}{m_{i}}\right]
$$

This implies that $a_{j}=b_{j}$ for $j>i$.

Analogously, the condition $\left[\frac{a}{m_{i}}\right] \neq\left[\frac{b}{m_{i}}\right]$ means that $a_{j} \neq b_{j}$ for some $j>i$.

The conditions $\left[\frac{a}{m_{i}}\right]=\left[\frac{b}{m_{i}}\right],\left[\frac{a}{m_{i-1}}\right] \neq\left[\frac{b}{m_{i-1}}\right]$ taken together mean that $a_{i} \neq b_{i}$, which implies $|l(a)-l(b)|=m_{i}$. We get that the matrix element of the Parisi matrix $Q_{a b}$ depends only on the ultrametric distance in $G$ of the indices $|l(a)-l(b)|$ : if $|l(a)-l(b)|$ equals to $m_{i}$, then $Q_{a b}=q_{i}$, which implies the theorem.

\section{New family of replica block matrices}

In the present section we build a new family of replica matrices, which generalize the Parisi matrices (22), used in the replica approach [20, [21], 22. These matrices will be some kind of block matrices, and each of the constructed block matrices will correspond to some (directed) tree.

We call a partially ordered set directed (and the corresponding partial order a direction), if an arbitrary finite subset of the partially ordered set has the unique supremum. We consider directed trees, which define ultrametric spaces (see the Appendix for details).

Consider a finite directed tree of the following form: we have the vertex $I$, called the root of the tree. Then we have $p_{I}$, where $p_{I} \geq 2$, vertices $I_{j}, j=0, \ldots, p_{I}-1$, which are connected by edges to the vertex $I$ and the partial order is defined as $I_{j}<I$. Then, for (some, probably not for all) vertices $J=I_{j}$ we have $p_{J}$ vertices $J_{j}, j=0, \ldots, p_{J}-1$, which are connected by edges to the vertex $J$ and the partial order is defined as $J_{j}<J$. Note that the branching indices $p_{J}$, corresponding to different $J=I_{j}$ are not necessarily equal. Then we iterate this procedure finite number of times (again, we may take iteration not for all obtained vertices, but only for some particular vertices), and obtain the finite directed tree $\mathcal{S}$. We can obtain an arbitrary finite tree in this way (but not an arbitrary finite directed tree).

Let us construct $n \times n$ block matrix $Q$, where $n$ is the number of elements in the set $\mathcal{S}_{\text {min }}$ of minimal elements in the tree $\mathcal{S}$. Enumerate minimal vertices $i \in \mathcal{S}_{\min }$ by 
$i=1, \ldots, n$. This index will enumerate the rows and columns of the block matrix $Q$. To each of the vertices $J \in \mathcal{S} \backslash \mathcal{S}_{\text {min }}$ we put into correspondence a number $q_{J}$.

Then we define the block matrix $Q$, for which the diagonal matrix elements are zero:

$$
Q_{i i}=0
$$

and the matrix element $Q_{i j}, i \neq j, i, j=1, \ldots, n$, is defined using the partial order in the tree $\mathcal{S}$ :

$$
Q_{i j}=q_{\sup (i, j)} \sqrt{\mu_{i} \mu_{j}}, \quad i \neq j
$$

where $\sup (i, j)$ is the vertex in $\mathcal{S}$ which is the supremum with respect to the partial order in $\mathcal{S}$ of the minimal vertices with the numbers $i$ and $j$ (the supremum of two vertices is the smallest vertex, which is larger both of the vertices). Here $q_{\sup (i, j)}$ is the mentioned above number, which corresponds to the vertex $\sup (i, j)$, and $\mu_{i}, \mu_{j}$ are some positive numbers (equal to measures of balls in some ultrametric space), see the definition in the next section.

Formula (5), which allows to describe the introduced replica block matrices in terms of directed trees is the crucial ingredient in the approach of the present paper. This formula can be considered as a natural generalization of $p$-adic parameterization of the Parisi matrix (2) onto the case of general ultrametric spaces.

Consider examples of the introduced replica matrices.

Example 1 The simplest example of the matrix of the described in the present section form (which differs from the Parisi matrix) is

$$
Q_{1}=\left(\begin{array}{ccccc}
0 & q_{1} & q_{0} & q_{0} & q_{0} \\
q_{1} & 0 & q_{0} & q_{0} & q_{0} \\
q_{0} & q_{0} & 0 & q_{2} & q_{2} \\
q_{0} & q_{0} & q_{2} & 0 & q_{2} \\
q_{0} & q_{0} & q_{2} & q_{2} & 0
\end{array}\right)
$$

Here we have two blocks, of the sizes $2 \times 2$ and $3 \times 3$, which are combined in the $2 \times 2$ block matrix with the described blocks at the diagonal. The resulting dimension of the matrix is $5 \times 5$.

By the procedure, described at the present section, it corresponds to the directed tree with the vertices, enumerated as $0,1,2,10,11,20,21,22$. The partial order on the vertices is defined as follows:

$$
0>1, \quad 0>2, \quad 1>10, \quad 1>11, \quad 2>20, \quad 2>21, \quad 2>22
$$

The pairs of ordered vertices listed above are connected by (directed) edges.

The vertices of the tree correspond to the matrix elements of the matrix as follows. The vertex 0 correspond to the value $q_{0}$, the vertex 1 correspond to the value $q_{1}$, the vertex 2 correspond to the value $q_{2}$. The lowest vertices $10,11,20,21$, 22 enumerate the rows and the columns of the matrix (in the above order). The vertices 1 and 2 enumerate the blocks, and one can say that the vertex 0 enumerate the matrix itself. 
Example 2 The second more complicated example of the block matrix is

$$
Q_{2}=\left(\begin{array}{ccccccc}
0 & q_{3} & q_{1} & q_{1} & q_{1} & q_{0} & q_{0} \\
q_{3} & 0 & q_{1} & q_{1} & q_{1} & q_{0} & q_{0} \\
q_{1} & q_{1} & 0 & q_{4} & q_{4} & q_{0} & q_{0} \\
q_{1} & q_{1} & q_{4} & 0 & q_{4} & q_{0} & q_{0} \\
q_{1} & q_{1} & q_{4} & q_{4} & 0 & q_{0} & q_{0} \\
q_{0} & q_{0} & q_{0} & q_{0} & q_{0} & 0 & q_{2} \\
q_{0} & q_{0} & q_{0} & q_{0} & q_{0} & q_{2} & 0
\end{array}\right)
$$

Here we have two blocks, of the sizes $2 \times 2$ and $5 \times 5$, which are combined in the $7 \times 7$ block matrix with the mentioned blocks at the diagonal. The $5 \times 5$ block is the block matrix $Q_{1}$ from the previous example (which itself contains nontrivial blocks).

\section{Ultrametric PDO and replica matrices}

In the present section we show that the introduced block matrices are related to ultrametric pseudodifferential operators (PDO) on ultrametric spaces, considered in [6, 7], see the Appendix for discussion. The construction of ultrametric spaces and the corresponding PDO is based on a directed tree, which we will denote $\mathcal{T}$. Consider a subtree $\mathcal{S} \subset \mathcal{T}$ satisfying the following definition.

Definition 3 The subset $\mathcal{S}$ in a directed tree $\mathcal{T}$ (with the partial order of the kind considered in the Appendix) is called of the analytic type, iff:

1) $\mathcal{S}$ is finite;

2) $\mathcal{S}$ is a directed subtree in $\mathcal{T}$ (where the direction in $\mathcal{S}$ is the restriction of the direction in $\mathcal{T}$ onto $\mathcal{S}$ );

3) The directed subtree $\mathcal{S}$ obey the following property: if $\mathcal{S}$ contains a vertex $I$ and a vertex $J: J<I,|I J|=1$, then the subtree $\mathcal{S}$ contains all the vertices $L$ in $\mathcal{T}: L<I,|I L|=1$.

The maximal vertex in $\mathcal{S}$ we will denote $K$.

Absolute of the directed tree $\mathcal{T}$, see [6], 7] and the Appendix for the details, is the ultrametric space $X(\mathcal{T})$ with the naturally defined measure $\mu$. Vertices of the tree $\mathcal{T}$ are in one to one correspondence with balls (or disks) in $X(\mathcal{T})$. We denote by $J$ the disk in $X(\mathcal{T})$, corresponding to vertex $J$, and by $\chi_{J}$ we denote the characteristic function of this disk.

For the finite subtree $\mathcal{S} \subset \mathcal{T}$ of the analytic type consider the space $D(\mathcal{S})^{1}$, which is the linear span of vectors $\chi_{J}$ with $J \in \mathcal{S}$. We consider this space as the subspace in the space $L^{2}(X, \mu)$ of quadratically integrable with respect to the measure $\mu$ functions on the absolute. Obviously in the space $D(\mathcal{S})$ there is the

\footnotetext{
${ }^{1}$ The space $D(\mathcal{S})$ is a finite dimensional space of test functions on the ultrametric space $X$ and is an analogue of the Bruhat-Schwartz space of test functions of $p$-adic argument. The space $D(X)$ of test functions on the absolute is the inductive limit of the spaces $D(\mathcal{S}): D(X)=\lim _{\operatorname{ind}} \rightarrow \mathcal{T} D(\mathcal{S})$.
} 
orthonormal basis $\left\{\frac{\chi_{J}}{\sqrt{\mu(J)}}\right\}$ with $J$ running over the minimal vertices in $\mathcal{S}$, i.e. a vector $f$ in $D(\mathcal{S})$ can be put into the form

$$
f=\sum_{J \in \mathcal{S}_{\min }} f_{J} \frac{\chi_{J}}{\sqrt{\mu(J)}}
$$

where the summation runs over minimal elements in $\mathcal{S}$.

Consider the operator $Q(\mathcal{S})=\Pi(\mathcal{S}) T$ in $D(\mathcal{S})$, where $\Pi(\mathcal{S})$ is the orthogonal projection in the space $L^{2}(X, \mu)$ onto $D(\mathcal{S})$, and $T$ is an ultrametric pseudodifferential operator $(\mathrm{PDO})$, see the Appendix. Operator $Q(\mathcal{S})$ is an operator in finite dimensional linear space $D(\mathcal{S})$.

Lemma 4 Action of $Q(\mathcal{S})=\Pi(\mathcal{S}) T$ on the functions (8) in $D(\mathcal{S})$ is given by the formula

$$
(Q(\mathcal{S}) f)_{I}=\sum_{J \in \mathcal{S}_{\min }} \sqrt{\mu(I) \mu(J)} T(\sup (I, J))\left[\sqrt{\frac{\mu(J)}{\mu(I)}} f_{I}-f_{J}\right]+C_{\mathcal{S}} f_{I}
$$

where

$$
C_{\mathcal{S}}=\int_{y \notin K} d \mu(y) T(I, y)=\sum_{L>K} T(L) \mu(L)\left(1-p_{L}\right)^{-1}
$$

and $I, J$ run over the minimal vertices in $\mathcal{S}$.

Proof Take $I \in \mathcal{S}_{\text {min }}$ and consider the scalar product in $L^{2}(X, \mu)$ :

$$
\begin{gathered}
\left\langle\frac{\chi_{I}}{\sqrt{\mu(I)}}, T \sum_{J \in \mathcal{S}_{\min }} f_{J} \frac{\chi_{J}}{\sqrt{\mu(J)}}\right\rangle= \\
=\sum_{J \in \mathcal{S}_{\min }} \frac{1}{\sqrt{\mu(I) \mu(J)}} f_{J} \int_{x \in I} d \mu(x) \int d \mu(y) T(x, y)\left(\chi_{J}(x)-\chi_{J}(y)\right)= \\
=\frac{1}{\mu(I)} f_{I} \int_{x \in I} d \mu(x) \int d \mu(y) T(x, y)\left(\chi_{I}(x)-\chi_{I}(y)\right)+ \\
+\sum_{J \in \mathcal{S}_{\min }, J \neq I} \frac{1}{\sqrt{\mu(I) \mu(J)}} f_{J} \int_{x \in I} d \mu(x) \int d \mu(y) T(x, y)\left(\chi_{J}(x)-\chi_{J}(y)\right)= \\
-\sum_{J \in \mathcal{S}_{\min }, J \neq I} \frac{1}{\mu(I)} f_{I} \int_{x \in I} d \mu(x) \int_{y \notin I} d \mu(y) T(x, y)- \\
=f_{I} \int_{y \notin I} d \mu(y) T(I, y)-\sum_{J \in \mathcal{S}_{\min }, J \neq I} \sum_{J \in \mathcal{S}_{\min }, J \neq I} \mu(J) T(\sup (I, J))- \\
=f_{I} \int_{y \notin K} d \mu(y) T(I, y)+f_{I} \sum_{x \in I} d \mu(x) \int d \mu(y) \chi_{J}(y)=
\end{gathered}
$$




$$
\begin{gathered}
-\sum_{J \in \mathcal{S}_{\min }, J \neq I} \sqrt{\mu(I) \mu(J)} T(\sup (I, J)) f_{J}= \\
=f_{I} \int_{y \notin K} d \mu(y) T(I, y)+\sum_{J \in \mathcal{S}_{\min }} \sqrt{\mu(I) \mu(J)} T(\sup (I, J))\left[\sqrt{\frac{\mu(J)}{\mu(I)}} f_{I}-f_{J}\right] .
\end{gathered}
$$

This proves the lemma.

Therefore, the operator $Q(\mathcal{S})$ corresponds to the block matrix (5):

$$
Q_{I J}=\sqrt{\mu(I) \mu(J)} T(\sup (I, J)), \quad I, J \in \mathcal{S}_{\text {min }}
$$

defined at the previous section. The lemma above shows that this matrix is related to the restriction of action of the ultrametric PDO onto the space $D(\mathcal{S})$, corresponding to the subtree $S \subset \mathcal{T}$ of the analytic type. In the following we will omit the index $\mathcal{S}$ in the notation of the replica matrix $Q(\mathcal{S})$, i.e. we will write $Q$ instead of $Q(\mathcal{S})$.

Remark It is easy to construct the generalization of the Parisi anzats on the case of general ultrametric spaces. The generalization of the Parisi matrix will look like

$$
Q_{I J}=\sqrt{\mu(I) \mu(J)} F(\mu((\sup (I, J)))), \quad I, J \in \mathcal{S}_{\min }, \quad F(x) \geq 0,
$$

where $F$ is a non-negative function of real argument. Let us note that the matrix (9) is more general than the matrix (10), even in the $p$-adic case.

Remark Note that $T(I, y)$ in the expression for $C_{\mathcal{S}}$ does not depend on $I \in \mathcal{S}$ and depends only on $K=\sup \mathcal{S}$. The constant $C_{\mathcal{S}}$ tends to zero if the largest vertex of $\mathcal{S}$ tends to infinity. We will ignore the contribution containing $C_{\mathcal{S}}$ in the following.

\section{Computations with replica matrices}

In the present section we compute the products and traces of the degrees for the introduced in the previous section matrices. Some similar computations for $p$-adic case can be found in [4]. The next lemma gives some useful formulas for computations, related to subtrees of analytic type.

\section{Lemma 5}

$$
\begin{gathered}
\sum_{J \in \mathcal{S}_{\min }: \sup (I, J)=L} \mu(J)=\mu(L)\left(1-p_{L}^{-1}\right) \\
\sum_{I, J \in \mathcal{S}_{\min }: \sup (I, J)=L} \mu(I) \mu(J)=\mu^{2}(L)\left(1-p_{L}^{-1}\right)
\end{gathered}
$$

Proof of the lemma is by additivity of the measure $\mu$.

The following lemma computes the important in the replica approach functional $\operatorname{tr} Q=\sum_{a \neq b} Q_{a b}$. 


\section{Lemma 6}

$$
\sum_{I, J \in \mathcal{S}_{\min } ; I \neq J} \mu(I) \mu(J) T(\sup (I, J))=\sum_{J \in \mathcal{S} \backslash \mathcal{S}_{\min }} T(J) \mu^{2}(J)\left(1-p_{J}^{-1}\right)
$$

Proof Computing the sum over $J$ we get

$$
\sum_{I, J \in \mathcal{S}_{\min } ; I \neq J} \mu(I) \mu(J) T(\sup (I, J))=\sum_{I \in \mathcal{S}_{\min }} \mu(I) \sum_{I<L \leq K} T(L) \sum_{J \in \mathcal{S}_{\min }: \sup (I, J)=L} \mu(J)
$$

where $K$ is the largest vertex in $\mathcal{S}$. This reduces to

$$
\begin{gathered}
\sum_{I \in \mathcal{S}_{\min }} \mu(I) \sum_{I<L \leq K} T(L) \mu(L) \\
\left(1-p_{L}^{-1}\right)=\sum_{J \in \mathcal{S} \backslash \mathcal{S}_{\min }} T(J) \mu(J)\left(1-p_{J}^{-1}\right) \sum_{I \in \mathcal{S}_{\min }: I<J} \mu(I)= \\
=\sum_{J \in \mathcal{S} \backslash \mathcal{S}_{\min }} T(J) \mu^{2}(J)\left(1-p_{J}^{-1}\right)
\end{gathered}
$$

This finishes the proof of the lemma.

Prove the following important lemma, which allows to compute products of replica matrices in terms of summation over the paths in corresponding trees.

Lemma 7 For $I, J \in \mathcal{S}_{\min }$ one has: if $I \neq J$, then

$$
\begin{gathered}
\sum_{L \in \mathcal{S}_{\min }, L \neq I, J} \mu(L) T_{1}(\sup (I, L)) T_{2}(\sup (L, J))= \\
=\mu(\sup (I, J))\left(1-2 p_{\sup (I, J)}^{-1}\right) T_{1}(\sup (I, J)) T_{2}(\sup (I, J))+ \\
+\sum_{L: \sup (I, J)<L \leq K} \mu(L)\left(1-p_{L}^{-1}\right) T_{1}(L) T_{2}(L)+ \\
+\left[\sum_{L: I<L<\sup (I, J)} \mu(L)\left(1-p_{L}^{-1}\right) T_{1}(L)\right] T_{2}(\sup (I, J))+ \\
+T_{1}\left(\sup _{\sup (I, J))}\left[\sum_{L: J<L<\sup (I, J)} \mu(L)\left(1-p_{L}^{-1}\right) T_{2}(L)\right]\right.
\end{gathered}
$$

and for $I=J$ one has

$$
\sum_{L \in \mathcal{S}_{\min }, L \neq I} \mu(L) T_{1}(\sup (I, L)) T_{2}(\sup (L, I))=\sum_{L: I<L \leq K} \mu(L)\left(1-p_{L}^{-1}\right) T_{1}(L) T_{2}(L)
$$

In particular

$$
\sum_{I, J \in \mathcal{S}_{\min } ; I \neq J} \mu(I) \mu(J) T(\sup (I, J))^{2}=\sum_{J \in \mathcal{S} \backslash \mathcal{S}_{\min }} T(J)^{2} \mu^{2}(J)\left(1-p_{J}^{-1}\right)
$$


Proof $\quad$ Compute for $I \neq J$

$$
\begin{gathered}
\sum_{L \in \mathcal{S}_{\min }, L \neq I, J} \mu(L) T_{1}(\sup (I, L)) T_{2}(\sup (L, J))= \\
=\sum_{L \in \mathcal{S}_{\min }, L \neq I, J: \sup (L, \sup (I, J))>\sup (I, J)} \mu(L) T_{1}(\sup (I, L)) T_{2}(\sup (L, J))+ \\
+\sum_{L \in \mathcal{S}_{\min }, L \neq I, J: \sup (L, I)<\sup (I, J)} \mu(L) T_{1}(\sup (I, L)) T_{2}(\sup (L, J))+ \\
+\sum_{L \in \mathcal{S}_{\min }, L \neq I, J: \sup (L, J)<\sup (I, J)} \mu(L) T_{1}(\sup (I, L)) T_{2}(\sup (L, J))+ \\
+\sum_{L \in \mathcal{S}_{\min ,}, L \neq I, J: \sup (L, I)=\sup (L, J)=\sup (I, J)} \mu(L) T_{1}(\sup (I, L)) T_{2}(\sup (L, J)) \\
\quad=\sum_{L: \sup (I, J)<L \leq K} \mu(L)\left(1-p_{L}^{-1}\right) T_{1}(L) T_{2}(L)+ \\
+\sum_{L: I<L<\sup (I, J)} \mu(L)\left(1-p_{L}^{-1}\right) T_{1}(L) T_{2}(\sup (I, J))+ \\
+\sum_{L: J<L<\sup (I, J)} \mu(L)\left(1-p_{L}^{-1}\right) T_{1}(\sup (I, J)) T_{2}(L)+ \\
+\mu(\sup (I, J))\left(1-2 p_{\sup (I, J)}^{-1}\right) T_{1}(\sup (I, J)) T_{2}(\sup (I, J))
\end{gathered}
$$

Analogously, for $I=J$ one obtains

$$
\sum_{L \in \mathcal{S}_{\min }, L \neq I} \mu(L) T_{1}(\sup (I, L)) T_{2}(\sup (L, I))=\sum_{L: I<L \leq K} \mu(L)\left(1-p_{L}^{-1}\right) T_{1}(L) T_{2}(L)
$$

This finishes the proof of the lemma.

The next lemma gives the trace of the cubic combination of the replica matrices.

Lemma 8 For the trace of the cubic combination of the replica block matrices we get

$$
\begin{gathered}
\operatorname{tr} Q_{1} Q_{2} Q_{3}= \\
=\sum_{A, B, C \in \mathcal{S}_{\min }, A \neq B, B \neq C, C \neq A} \mu(A) \mu(B) \mu(C) T_{1}(\sup (A, B)) T_{2}(\sup (B, C)) T_{3}(\sup (C, A))= \\
=\sum_{L \in \mathcal{S} \backslash \mathcal{S}_{\min }} \mu^{3}(L)\left(1-p_{L}^{-1}\right)\left(1-2 p_{L}^{-1}\right) T_{1}(L) T_{2}(L) T_{3}(L)+ \\
+\sum_{L \in \mathcal{S} \backslash \mathcal{S}_{\min }} T_{1}(L) T_{2}(L) \mu(L)\left(1-p_{L}^{-1}\right)\left[\sum_{B \in \mathcal{S} \backslash \mathcal{S}_{\min }: B<L} \mu^{2}(B)\left(1-p_{B}^{-1}\right) T_{3}(B)\right]+ \\
+\sum_{L \in \mathcal{S} \backslash \mathcal{S}_{\min }} T_{2}(L) T_{3}(L) \mu(L)\left(1-p_{L}^{-1}\right)\left[\sum_{B \in \mathcal{S} \backslash \mathcal{S}_{\min }: B<L} \mu^{2}(B)\left(1-p_{B}^{-1}\right) T_{1}(B)\right]+ \\
+\sum_{L \in \mathcal{S} \backslash \mathcal{S}_{\min }} T_{3}(L) T_{1}(L) \mu(L)\left(1-p_{L}^{-1}\right)\left[\sum_{B \in \mathcal{S} \backslash \mathcal{S}_{\min }: B<L} \mu^{2}(B)\left(1-p_{B}^{-1}\right) T_{2}(B)\right]
\end{gathered}
$$


Remark In more compact form the above formula can be written as

$$
\begin{gathered}
\sum_{A, B, C \in \mathcal{S}_{\min }, A \neq B, B \neq C, C \neq A} \mu(A) \mu(B) \mu(C) T_{1}(\sup (A, B)) T_{2}(\sup (B, C)) T_{3}(\sup (C, A))= \\
=\sum_{L \in \mathcal{S} \backslash \mathcal{S}_{\min }} \mu^{3}(L)\left(1-p_{L}^{-1}\right)\left(1-2 p_{L}^{-1}\right) T_{1}(L) T_{2}(L) T_{3}(L)+ \\
\quad+\sum_{L \in \mathcal{S} \backslash \mathcal{S}_{\min }} \mu(L)\left(1-p_{L}^{-1}\right) \sum_{B \in \mathcal{S} \backslash \mathcal{S}_{\min }: B<L} \mu^{2}(B)\left(1-p_{B}^{-1}\right) \\
{\left[T_{1}(L) T_{2}(L) T_{3}(B)+T_{2}(L) T_{3}(L) T_{1}(B)+T_{3}(L) T_{1}(L) T_{2}(B)\right]}
\end{gathered}
$$

The property of the sum in (12), that two of the three (or even three of the three) indices $I$ of the coefficients $T(I)$ coincide, and the two coinciding indices are larger than the third, is related to the fact that in ultrametric space all triangles are equilateral with equal larger edges. The analogous observations for the Parisi RSB ansatz were made in [1]. Note that here (unlike in [1]) we did not yet introduce any kind of the $n \rightarrow 0$ limit.

Proof of Lemma $8 \quad$ Compute

$\sum_{A, B, C \in \mathcal{S}_{\text {min }}, A \neq B, B \neq C, C \neq A} \mu(A) \mu(B) \mu(C) T_{1}(\sup (A, B)) T_{2}(\sup (B, C)) T_{3}(\sup (C, A))=$

$$
\begin{aligned}
& =\sum_{A, C \in \mathcal{S}_{\min } ; C \neq A} \mu(A)\left[\sum_{B: \sup (A, C)<B \leq K} \mu(B)\left(1-p_{B}^{-1}\right) T_{1}(B) T_{2}(B)+\right. \\
& +\mu(\sup (A, C))\left(1-2 p_{\sup (A, C)}^{-1}\right) T_{1}(\sup (A, C)) T_{2}(\sup (A, C))+ \\
& \quad+\left[\sum_{B: A<B<\sup (A, C)} \mu(B)\left(1-p_{B}^{-1}\right) T_{1}(B)\right] T_{2}(\sup (A, C))+ \\
& \left.+T_{1}(\sup (A, C))\left[\sum_{B: C<B<\sup (A, C)} \mu(B)\left(1-p_{B}^{-1}\right) T_{2}(B)\right]\right] \mu(C) T_{3}(\sup (C, A))
\end{aligned}
$$

Discuss the third contribution to the above sum, i.e. the expression

$$
\sum_{A, C \in \mathcal{S}_{\min } ; C \neq A} \mu(A) \mu(C) T_{2}(\sup (A, C)) T_{3}(\sup (C, A))\left[\sum_{B: A<B<\sup (A, C)} \mu(B)\left(1-p_{B}^{-1}\right) T_{1}(B)\right]
$$

In this expression we put the summation as the following composition of the three summations

$$
\sum_{A, C \in \mathcal{S}_{\min } ; C \neq A}=\sum_{C \in \mathcal{S}_{\min }} \sum_{L: C<L \leq K} \sum_{A \in \mathcal{S}_{\min }: \sup (A, C)=L}
$$

This implies

$$
\sum_{C \in \mathcal{S}_{\min }} \sum_{L: C<L \leq K} \sum_{A \in \mathcal{S}_{\min }: \sup (A, C)=L} \mu(A) \mu(C) T_{2}(\sup (A, C)) T_{3}(\sup (C, A))
$$




$$
\begin{aligned}
& {\left[\sum_{B: A<B<\sup (A, C)} \mu(B)\left(1-p_{B}^{-1}\right) T_{1}(B)\right]=} \\
= & \sum_{C \in \mathcal{S}_{\min }} \mu(C) \sum_{L: C<L \leq K} T_{2}(L) T_{3}(L) \sum_{A \in \mathcal{S}_{\min }: \sup (A, C)=L} \mu(A)\left[\sum_{B: A<B<L} \mu(B)\left(1-p_{B}^{-1}\right) T_{1}(B)\right]= \\
= & \sum_{C \in \mathcal{S}_{\min }} \mu(C) \sum_{L: C<L \leq K} T_{2}(L) T_{3}(L)\left[\sum_{B \in \mathcal{S} \backslash \mathcal{S}_{\min }: B<L, \sup (B, C)=L} \mu^{2}(B)\left(1-p_{B}^{-1}\right) T_{1}(B)\right]= \\
= & \sum_{L \in \mathcal{S} \backslash \mathcal{S}_{\min }} T_{2}(L) T_{3}(L) \sum_{C \in \mathcal{S}_{\min }: C<L} \mu(C)\left[\sum_{B \in \mathcal{S} \backslash \mathcal{S}_{\min }: B<L, \sup (B, C)=L} \mu^{2}(B)\left(1-p_{B}^{-1}\right) T_{1}(B)\right]= \\
= & \sum_{L \in \mathcal{S} \backslash \mathcal{S}_{\min }} T_{2}(L) T_{3}(L) \mu(L)\left(1-p_{L}^{-1}\right)\left[\sum_{B \in \mathcal{S} \backslash \mathcal{S}_{\min }: B<L} \mu^{2}(B)\left(1-p_{B}^{-1}\right) T_{1}(B)\right]
\end{aligned}
$$

Analogously, the fourth contribution

$$
\sum_{A, C \in \mathcal{S}_{\min } ; C \neq A} \mu(A) \mu(C) T_{1}(\sup (A, C)) T_{3}(\sup (C, A))\left[\sum_{B: C<B<\sup (A, C)} \mu(B)\left(1-p_{B}^{-1}\right) T_{2}(B)\right]
$$

will give

$$
\sum_{L \in \mathcal{S} \backslash \mathcal{S}_{\min }} T_{1}(L) T_{3}(L) \mu(L)\left(1-p_{L}^{-1}\right)\left[\sum_{B \in \mathcal{S} \backslash \mathcal{S}_{\min }: B<L} \mu^{2}(B)\left(1-p_{B}^{-1}\right) T_{2}(B)\right]
$$

Compute the first contribution. We get

$$
\begin{aligned}
& \sum_{A, C \in \mathcal{S}_{\min } ; C \neq A} \mu(A) \mu(C) T_{3}(\sup (C, A))\left[\sum_{B: \sup (A, C)<B \leq K} \mu(B)\left(1-p_{B}^{-1}\right) T_{1}(B) T_{2}(B)\right]= \\
= & \sum_{B \in \mathcal{S} \backslash \mathcal{S}_{\text {min }}} \mu(B)\left(1-p_{B}^{-1}\right) T_{1}(B) T_{2}(B)\left[\sum_{A, C \in \mathcal{S}_{\text {min }} ; C \neq A ; A, C<B} \mu(A) \mu(C) T_{3}(\sup (C, A))\right]= \\
= & \sum_{B \in \mathcal{S} \backslash \mathcal{S}_{\text {min }}} \mu(B)\left(1-p_{B}^{-1}\right) T_{1}(B) T_{2}(B)\left[\sum_{L \in \mathcal{S} \backslash \mathcal{S}_{\min }: L<B} \mu^{2}(L)\left(1-p_{L}^{-1}\right) T_{3}(L)\right]
\end{aligned}
$$

The second contribution reduces to

$$
\begin{aligned}
& \sum_{A, C \in \mathcal{S}_{\min } ; C \neq A} \mu(A) \mu(C) \mu(\sup (A, C))\left(1-2 p_{\sup (A, C)}^{-1}\right) \\
& T_{1}(\sup (A, C)) T_{2}(\sup (A, C)) T_{3}(\sup (C, A))= \\
= & \sum_{L \in \mathcal{S} \backslash \mathcal{S}_{\min }} \mu^{3}(L)\left(1-p_{L}^{-1}\right)\left(1-2 p_{L}^{-1}\right) T_{1}(L) T_{2}(L) T_{3}(L)
\end{aligned}
$$

Combining formulas (13)-(16), we obtain the proof of the lemma.

The next lemma gives the trace of the quartic combination. 


\section{Lemma 9}

$$
\begin{aligned}
& \operatorname{tr} Q^{4}=\sum_{A, B, C, D \in \mathcal{S}_{\min }, A \neq B, B \neq C, C \neq D, D \neq A} \mu(A) \mu(B) \mu(C) \mu(D) \\
& T(\sup (A, B)) T(\sup (B, C)) T(\sup (C, D)) T(\sup (D, A))= \\
& =\sum_{L \in \mathcal{S} \backslash \mathcal{S}_{\min }} \mu^{4}(L)\left(1-p_{L}^{-1}\right)\left(1-2 p_{L}^{-1}\right)^{2} T(L)^{4}+ \\
& +2 \sum_{L \in \mathcal{S} \backslash \mathcal{S}_{\min }} \mu^{3}(L)\left(1-p_{L}^{-1}\right)\left(1-2 p_{L}^{-1}\right) T(L)^{2} \sum_{M: L<M \leq K} \mu(M)\left(1-p_{M}^{-1}\right) T(M)^{2}+ \\
& +4 \sum_{L \in \mathcal{S} \backslash \mathcal{S}_{\min }} \mu(L)\left(1-2 p_{L}^{-1}\right) T(L)^{3}\left[\sum_{M \in \mathcal{S} \backslash \mathcal{S}_{\min }: M<L} \mu^{3}(M)\left(1-p_{M}^{-1}\right)^{2} T(M)\right]+ \\
& +\sum_{M \in \mathcal{S} \backslash \mathcal{S}_{\min }} \mu^{2}(M)\left(1-p_{M}^{-1}\right)\left[\sum_{L: M<L \leq K} \mu(L)\left(1-p_{L}^{-1}\right) T(L)^{2}\right]^{2}+ \\
& +4 \sum_{I \in \mathcal{S} \backslash \mathcal{S}_{\min }}\left[\sum_{M \in \mathcal{S} \backslash \mathcal{S}_{\min }: M<I} \mu^{3}(M)\left(1-p_{M}^{-1}\right)^{2} T(M)\right] T(I)\left[\sum_{L: I<L \leq K} \mu(L)\left(1-p_{L}^{-1}\right) T(L)^{2}\right] \\
& +2 \sum_{L \in \mathcal{S} \backslash \mathcal{S}_{\min }}\left[\sum_{M \in \mathcal{S} \backslash \mathcal{S}_{\min }: M<L} \mu^{4}(M)\left(1-p_{M}^{-1}\right)^{3} T(M)^{2}\right] T(L)^{2}+ \\
& +4 \sum_{L \in \mathcal{S} \backslash \mathcal{S}_{\min }}\left[\sum_{M \in \mathcal{S} \backslash \mathcal{S}_{\min }: M<L} \mu^{3}(M)\left(1-p_{M}^{-1}\right)^{2} T(M) \sum_{I: M<I<L} \mu(I)\left(1-p_{I}^{-1}\right) T(I)\right] T(L)^{2}+ \\
& +\sum_{L, M \in \mathcal{S} \backslash \mathcal{S}_{\min }} \mu^{2}(L) \mu^{2}(M)\left(1-p_{L}^{-1}\right)\left(1-p_{M}^{-1}\right) T(L) T(M) T(\sup (L, M))^{2}
\end{aligned}
$$

The proof is by application of lemmas 7,5 ,

\section{Comparison with the Parisi case}

In the present section we compare the computed in the previous section functionals of the introduced replica matrices with the known results for the Parisi matrices. For the Parisi anzats the corresponding tree looks as follows: the branching index $p_{j}$ depends on the integer $j$ (the level in the tree) for $j=1, \ldots, k, \mu(J)=m_{j}$, where

$$
m_{j}=\prod_{l=1}^{j} p_{l}, \quad m_{0}=1 .
$$

Values $T(J)$ of the function on a tree, in the case when the function $T(J)$ depends only on the level $j$, are encoded into $t^{(j)}$. 
Lemma 10 Under the described above conditions the computed in the previous section functionals of replica matrices reduce to the functionals of the Parisi anzats as follows:

$$
\begin{gathered}
J \in \mathcal{S} \backslash \mathcal{S}_{\min } \mapsto j=1, \ldots, k, \quad \mu(J) \mapsto m_{j}, \quad T(J) \mapsto t^{(j)}, \\
\sum_{J \in \mathcal{S} \backslash \mathcal{S}_{\min }} F(J) \mapsto \sum_{j=1}^{k} \frac{m_{k}}{m_{j}} f(j)
\end{gathered}
$$

In the sum above the coefficient $\frac{m_{k}}{m_{j}}$ corresponds to the multiplicity of vertices on the level $j$ of the tree.

Example 1 Compute the functional (111) of the form

$$
\sum_{I, J \in \mathcal{S}_{\min } ; I \neq J} \mu(I) \mu(J) T(\sup (I, J))=\sum_{J \in \mathcal{S} \backslash \mathcal{S}_{\min }} T(J) \mu^{2}(J)\left(1-p_{J}^{-1}\right)
$$

We get for (11), normalized by the division by $n=m_{k}=\mu(K)$ the following expression

$$
\sum_{j=1}^{k} t^{(j)} \frac{m_{k}}{m_{j}}\left[m_{j}-m_{j-1}\right] \frac{m_{j}}{m_{k}}=\sum_{j=1}^{k} t^{(j)}\left[m_{j}-m_{j-1}\right]
$$

where $\frac{m_{k}}{m_{j}}$ is the degeneracy of $t^{(j)}$, and $\frac{m_{j}}{m_{k}}$ is $\frac{\mu(J)}{\mu(K)}$. This reduces to the known answer of the Parisi replica symmetry breaking anzats

$$
\sum_{j=1}^{k} t^{(j)}\left[m_{j}-m_{j-1}\right]
$$

where $m_{0}=1$ and $m_{k}=n$.

Example 2 Compute the functional (12) (normalized by division by $n=\mu(K)$ ) of the form

$$
\begin{aligned}
& \frac{1}{\mu(K)} \sum_{L \in \mathcal{S} \backslash \mathcal{S}_{\min }} \mu^{3}(L)\left(1-p_{L}^{-1}\right)\left(1-2 p_{L}^{-1}\right) T_{1}(L) T_{2}(L) T_{3}(L)+ \\
& +\frac{1}{\mu(K)} \sum_{L \in \mathcal{S} \backslash \mathcal{S}_{\min }} \mu(L)\left(1-p_{L}^{-1}\right) \sum_{B \in \mathcal{S} \backslash \mathcal{S}_{\min }: B<L} \mu^{2}(B)\left(1-p_{B}^{-1}\right) \\
& {\left[T_{1}(L) T_{2}(L) T_{3}(B)+T_{2}(L) T_{3}(L) T_{1}(B)+T_{3}(L) T_{1}(L) T_{2}(B)\right]}
\end{aligned}
$$

We get

$$
\begin{gathered}
\frac{1}{m_{k}} \sum_{l=1}^{k} \frac{m_{k}}{m_{l}} m_{l}\left(m_{l}-m_{l-1}\right)\left(m_{l}-2 m_{l-1}\right) t_{1}^{(l)} t_{2}^{(l)} t_{3}^{(l)}+ \\
+\frac{1}{m_{k}} \sum_{l=1}^{k} \frac{m_{k}}{m_{l}}\left(m_{l}-m_{l-1}\right) \sum_{b=1}^{l-1} \frac{m_{l}}{m_{b}} m_{b}\left(m_{b}-m_{b-1}\right)\left[t_{1}^{(l)} t_{2}^{(l)} t_{3}^{(b)}+t_{2}^{(l)} t_{3}^{(l)} t_{1}^{(b)}+t_{3}^{(l)} t_{1}^{(l)} t_{2}^{(b)}\right]=
\end{gathered}
$$




$$
\begin{gathered}
=\sum_{l=1}^{k}\left(m_{l}-m_{l-1}\right)\left(m_{l}-2 m_{l-1}\right) t_{1}^{(l)} t_{2}^{(l)} t_{3}^{(l)}+ \\
+\sum_{l=1}^{k}\left(m_{l}-m_{l-1}\right) \sum_{b=1}^{l-1}\left(m_{b}-m_{b-1}\right)\left[t_{1}^{(l)} t_{2}^{(l)} t_{3}^{(b)}+t_{2}^{(l)} t_{3}^{(l)} t_{1}^{(b)}+t_{3}^{(l)} t_{1}^{(l)} t_{2}^{(b)}\right]
\end{gathered}
$$

This again fits into the standard RSB picture, replica computations of similar kind for the Parisi case were performed in [1].

\section{Appendix: trees, ultrametric spaces, PDO}

In the present section we, following papers [6], [7, define a family of ultrametric spaces related to trees. For discussion of relation between trees and ultrametric spaces see also 22]. An ultrametric space is a metric space with the metric $|x y|$ (the distance between $x$ and $y$ ), which satisfies the strong triangle inequality

$$
|a b| \leq \max (|a c|,|c d|), \quad \forall c
$$

We consider directed trees, i.e. trees with partial order, which is a direction. A partially ordered set is called directed (and the corresponding partial order - a direction), if an arbitrary finite subset has the unique supremum (remind that the supremum of the subset of a partially ordered set is a minimal element of the set, which is larger or equal to all elements of the subset).

Consider an arbitrary tree $\mathcal{T}$ (finite or infinite), such that the path in the tree between arbitrary two vertices is finite, and the number of edges incident to each of the vertices is finite. If a non-maximal vertex $I \in \mathcal{T}$ is incident to $p_{I}+1$ edges, we will say that the branching index of $I$ is $p_{I}$. If maximal index $I \in \mathcal{T}$ is incident to $p_{I}$ edges, we will say that the branching index of $I$ is $p_{I}$. Equivalently, branching index of a vertex $I$ in directed tree is the number of maximal elements, which less than $I$.

The absolute of a tree will be an ultrametric space (with respect to the naturally defined metric). Consider two equivalent definitions of the absolute of the tree.

The first definition is as follows. The infinitely continued path with the beginning in vertex $I$ is a path with the beginning in $I$, which is not a subset of a larger path with the beginning in $I$. The space of infinitely continued paths in the directed tree $\mathcal{T}$, which begin in some vertex $R$ (that is, the root) is called the absolute of the tree. Obviously the definition of the absolute of the tree does not depend on the choice of $R$ (taking any other vertex $A$ leads to an equivalent definition).

The equivalent definition of the absolute is as follows: the absolute is the space of equivalence classes of infinitely continued paths in the tree $\mathcal{T}$, such that any two paths in one equivalence class coincide starting from some vertex (i.e. the tails of the paths in one equivalence class are the same). If we choose in each of the equivalence classes the paths, which begin in vertex $R$, we will reproduce the first definition.

We consider trees with a partial order, where the partial order is defined in the following way. Fix the vertex $R$ and the point $\infty$ at the absolute. To fix the point $\infty$ at the absolute means to fix the infinitely continued path $R \infty$ from the vertex 
$R$ to $\infty$. The point $\infty$ we will call the infinite point, or the infinity. We define the following natural partial order on the set of vertices of the tree: $J>I$ if $J$ belongs to the path $I \infty$.

Consider the absolute with excluded infinite point, or equivalently, the space of equivalence classes of decreasing paths in $\mathcal{T}$. In the following we will call the absolute with excluded infinite point the absolute. We denote the absolute of the tree $\mathcal{T}$ by $X=X(\mathcal{T})$ (note that we already excluded the infinite point). Let us construct the ultrametric and the measure on $X$.

For the points $x, y$ of the absolute there exists a unique path $x y$ in the tree. The notation $x y$ should be understood in the following way. Since the points $x, y$ of the absolute are identified with the paths $R x$ and $R y$, the path $x y$ will be contained in $R x \cup R y$. Then there exists a unique vertex $A$ satisfying

$$
R x=R A x, \quad R y=R A y, \quad A x \bigcap A y=A
$$

The notation $A B C$ means that $A C=A B \cup B C$. Then

$$
x y=A x \bigcup A y
$$

Define the vertex in $\mathcal{T}$, which is the supremum of $x$ and $y$ :

$$
\sup (x, y)=x y \bigcap x \infty \bigcap y \infty
$$

Analogously, for vertices $A, B$ of the tree we define

$$
\sup (A, B)=A B \bigcap A \infty \bigcap B \infty
$$

as well as for $A \in \mathcal{T}, x \in X(\mathcal{T})$

$$
\sup (A, x)=A x \bigcap A \infty \bigcap x \infty
$$

Definitions (19), (20), (21) make $\mathcal{T}$ and $\mathcal{T} \cup X(\mathcal{T})$ the directed sets.

Put into correspondence to an edge in the tree the branching index of the largest vertex of the edge (this definition is correct, since any two vertices, connected by edge, are comparable). Then the distance $|x y|$ is introduced as the product of branching indices of edges in the directed path $R I, I=\sup (x, y)$ in the degrees \pm 1 , where branching indices of increasing edges are taken in the degree +1 , and branching indices of decreasing edges are taken in the degree -1 . Here an edge is called increasing, if the end of the edge is larger than the beginning, and is called decreasing in the opposite case:

$$
|x y|=\prod_{j=0}^{N-1} p_{I_{j} I_{j+1}}^{\varepsilon_{I_{j} I_{j+1}}}, \quad I_{0}=R, \ldots, I_{N}=I
$$

where $\varepsilon_{I_{j} I_{j+1}}=1$ for $I_{j}<I_{j+1}$, and $\varepsilon_{I_{j} I_{j+1}}=-1$ for $I_{j}>I_{j+1}$.

Lemma 11 The function $|x y|$ is an ultrametric (i.e. it is nonnegative, equal to zero only for $x=y$, symmetric, and satisfies the strong triangle inequality):

$$
|x y| \leq \max (|x z|,|y z|), \quad \forall z
$$


To define the measure $\mu$, it is enough to define this measure on the disks $I$, where disk $I$ is the set of all the infinitely continued paths incident to the vertex $I$ which intersect the path $I \infty$ only at the vertex $I$. Define the diameter $d_{I}$ of the disk as the supremum of the distance $|x y|$ between the paths $I x$ and $I y$ in $I$. Then $I$ is the ball of radius $d_{I}$ with its center on any of $I x \in I$.

Definition 12 The measure $\mu(I)$ of the disk $I$ is equal to the disk diameter.

Since the disk $I$ contains $p_{I}$ maximal subdisks, which by definitions of the ultrametric and the measure have the measure $p_{I}^{-1} \mu(I)$, the measure $\mu$ is additive on disks. By additivity we can extend the measure on algebra generated by disks ( $\sigma$-additivity of the measure will follow from the local compactness of the absolute, analogously to the case of the Lebesgue measure). We denote $L^{2}(X, \mu)$ the space of the square integrable (with respect to the defined measure) functions on the absolute.

Definition 13 We say that the operator in $L^{2}(X, \mu)$ of the form

$$
T f(x)=\int T(\sup (x, y))(f(x)-f(y)) d \mu(y)
$$

where $T(I)$ is a function on the tree $\mathcal{T}$, is an ultrametric pseudodifferential operator (PDO).

\section{Acknowledgements}

The authors would like to thank G.Parisi and I.V.Volovich for fruitful discussions and valuable comments. One of the authors (A.Kh.) would like to thank S.Albeverio for fruitful discussions and support of $p$-adic investigations. This paper has been partly supported by EU-Network "Quantum Probability and Applications". One of the authors (S.V.Kozyrev) has been partly supported by the CRDF (grant UM12421-KV-02), by The Russian Foundation for Basic Research (project 05-01-00884a), by the grant of the President of Russian Federation for the support of scientific schools NSh 1542.2003.1, by the Program of the Department of Mathematics of Russian Academy of Science "Modern problems of theoretical mathematics", by the grant of The Swedish Royal Academy of Sciences on collaboration with scientists of former Soviet Union, and by the grants DFG Project 436 RUS 113/809/0-1 and RFFI 05-01-04002-NNIO-a.

\section{References}

[1] M.Mezard, G.Parisi, N.Sourlas, G.Toulouse, M.Virasoro, Nature of the spin glass phase, Phys.Rev.Lett. 52(1984), N13, pp.1156-1159

[2] M.Mezard, G.Parisi, M.Virasoro, Spin-Glass Theory and Beyond, Singapore: World Scientific, 1987 
[3] V.A.Avetisov, A.Kh.Bikulov, S.V.Kozyrev, Application of $p$-adic analysis to models of spontaneous breaking of replica symmetry, Journal of Physics A, 1999, Vol.32, pp.8785-8791, http://xxx.lanl.gov/abs/cond-mat/9904360, Proceedings of the Conference "Quantum Theory: Reconsideration of Foundations", 17-21 June 2001, Växjö, Sweden, edited by A.Khrennikov, Växjö University Press 2002

[4] G.Parisi, N.Sourlas, $p$-Adic numbers and replica symmetry breaking, 2000 European Phys. J. B 14 535-542, http://xxx.lanl.gov/abs/cond-mat/9906095

[5] C.De Dominicis, D.M.Carlucci, T.Temesvari, Replica Fourier transform on ultrametric trees and block-diagonalizing multireplica matrices // J. Phys. I France. 1997. V. 7. P. 105-115. http://arxiv.org/abs/cond-mat/9703132

[6] A.Yu.Khrennikov, S.V.Kozyrev, Wavelets on ultrametric spaces, Applied and Computational Harmonic Analysis, Volume 19, 2005, P. 61-76

[7] A. Yu. Khrennikov and S. V. Kozyrev, Pseudodifferential operators on ultrametric spaces and ultrametric wavelets, Russian Math. Izv.. 2005. V.69.N.5. http://arxiv.org/abs/math-ph/0412062

[8] S.V.Kozyrev, Ultrametric pseudodifferential operators and wavelets for the case of non homogeneous measure, http://arxiv.org/abs/math-ph/0412082

[9] S.V.Kozyrev, Wavelet analysis as a $p$-adic spectral analysis // Russian Math. Izv. 2002. V. 66. N 2. P. 367. http://arxiv.org/abs/math-ph/0012019

[10] S.V.Kozyrev, $p$-Adic pseudodifferential operators and $p$-adic wavelets // Theor. Math. Physics. 2004. V. 138. N 3, P. 322-332. http://arxiv.org/abs/math-ph/0303045

[11] V.S.Vladimirov, I.V.Volovich, Ye.I.Zelenov, $p$-Adic analysis and mathematical physics. World Scientific, Singapore, 1994. Russian Edition: Moscow, Nauka, 1994

[12] I.V.Volovich, $p$-Adic String, Class. Quantum Gravity, 4(1987)L83-L87

[13] A.Khrennikov, $p$-Adic valued distributions in mathematical physics, Kluwer Academic Publ., Dordrecht, 1994

[14] A.Khrennikov, Non-Archimedean Analysis: Quantum Paradoxes, Dynamical Systems and Biological Models, Kluwer Academic Publishers, 1997

[15] A.Khrennikov, $p$-Adic discrete dynamical systems and their applications in physics and cognitive sciences, Russian Journal of Mathematical Physics, 11 (1), 2004, pp.45-70

[16] J.J.Benedetto, R.L.Benedetto, A wavelet theory for local fields and related groups, The Journal of Geometric Analysis, 14 (3), 2004, pp. 423-456 
[17] R.L.Benedetto, Examples of wavelets for local fields, http://arxiv.org/math.CA/abs/0312038

[18] A.N.Kochubei, Pseudo-Differential Equations and Stochastics over NonArchimedean Fields. New York: Marcel Dekker, 2001.

[19] V.A.Avetisov, A.Kh.Bikulov, S.V.Kozyrev, V.A.Osipov, p-Adic Models of Ultrametric Diffusion Constrained by Hierarchical Energy Landscapes // J. Phys. A: Math. Gen. 2002. V. 35. P. 177-189. http://arxiv.org/abs/cond-mat/0106506

[20] G.Parisi, Towards a mean field theory for spin glasses, Phys.Lett.A 73(1979)p.203-205

[21] G.Parisi, Infinite number of order parameters for spin glasses, Phys.Rev.Lett. 43(1979)p.1754-1756

[22] J.P.Serre, Trees. New York, Berlin: Springer Verlag, 1980. 